\title{
Quality of Service Support in Wireless Sensor Networks for Emergency Healthcare Services
}

\author{
Óscar Gama, Paulo Carvalho, J. A. Afonso, P. M. Mendes
}

\begin{abstract}
The reliable and efficient operation of emergency healthcare (e-emergency) services poses quality demands to the systems and underlying communication infrastructures. In this context, most existing wireless body sensor networks fall short in meeting these demands as they only offer an unreliable service delivery. e-Emergency systems must provide quality of service (QoS) support so that a pervasive and trustable assistance is provided to patients under health risk. This paper discusses the need for QoS in wireless e-health and e-emergency services. To demonstrate this need, some current and relevant ehealth projects with QoS requirements are presented. The study reveals the importance of providing QoS support in this emerging field of application and provides a summary characterizing the e-health proposals herein presented.
\end{abstract}

\section{INTRODUCTION}

A personal healthcare system consists of a group of sensors attached non-invasively to a patient, in order to monitor his/her vital signals to detect any life threatening abnormality. These body sensor networks (BSN) have been used in hospitals during the last decades using conventional wired equipment, hence not allowing the patient to move freely around. However, recent advances in wireless sensors technology are changing this scenario by permitting mobile and permanent monitoring of patients, even during their normal daily activities, and without compromising their quality of life [1]. In such healthcare systems, the sensed information at the patient's body is transmitted to a wireless base-station, located no more than a few tens of meters away, and then delivered to a remote diagnosis centre through a communication infrastructure.

Healthcare systems should be able to accomplish at least one crucial aim: to monitor a patient and, when an emergency occurs, to trigger immediately an event to alert the patient and/or to warn a remote caregiver. In this way, both the patient and the caregiver can take timely the right procedure in accordance with the clinical episode. In addition, the system should be able to trigger an alert anticipating the case where the patient is unaware of his/her health gravity.

Basically two main scenarios can be found in eemergency. In the first one, a patient is continuously

Manuscript received April 2, 2008

Oscar Gama and Paulo Carvalho are with the University of Minho, Informatics Dept., Braga, Portugal (emails: \{osg, pmc\}@di.uminho.pt).

J. A. Afonso and P. M. Mendes are with the University of Minho, Industrial Electronics Dept., Guimarães, Braga, Portugal (emails: \{jose.afonso, paulo.mendes\}@dei.uminho.pt). monitored indoors or outdoors to detect an eventual emergency situation requiring a prompt response from a physician. Recall that the first hour after a cardiac episode is critical for the success of the patient recovery, and hence is called golden hour. A patient could also be continuously monitored to detect, for instance, suspected cardiac arrhythmias.

Mass casualty disasters constitute the second scenario. Here, the main problem is the overwhelming number of patients that must be monitored and tracked by the caregivers. The ability to automate these tasks could greatly relieve the caregivers' workload, increase the quality and quantity of patient care, and help to detect patients requiring rapid intervention [2].

When a patient's clinical state turns from a non-critical situation into a critical one, a context change occurs and consequently the healthcare network should adapt its performance requirements to the new situation. For instance, higher monitoring activity and lower delay transmission of the vital signals might be required when the clinical situation of a patient changes from non-critical to critical. Hence, healthcare networks should provide QoS facilities for eemergency services, since these clearly demand for high reliability, guaranteed bandwidth, and short delays.

\section{VITAL SIGNALS MONITORING}

Emergency medical care requires monitoring of several vital signals simultaneously. For example, a patient in an ambulance is monitored for blood pressure, heart and respiration rates, and temperature. Besides these primary signals, other information may be captured to help diagnosis and medical decision, such as electrocardiogram (ECG), blood glucose level, blood oxygen saturation, heart and breathing sounds, or even an image in cases of trauma. All these data should be promptly accessible for comparison, computer aided analysis, and decision-making. However, to have a more encompassing picture of the clinical situation, the information about the patient's personal characteristics (risk factors, degree of disease, age, etc.) and environmental context (in bed or mobile, alone or not, at work or at home, etc.) should also be provided [3].

Table I presents the electrical characteristics of the vital signals usually used in emergency medical care $[4,5]$.

If some signal exceeds the threshold, the local supervisor node should trigger an alarm to inform a caregiver or the patient himself. Table II presents typical thresholds for 
$\mathrm{SpO}$, heart rate (HR), and blood pressure (BP) signals for alert detection [2].

TABLE I

VITAL SIGNAL ELECTRICAL CHARACTERISTICS

\begin{tabular}{|c|c|c|c|}
\hline $\begin{array}{c}\text { Vital signal } \\
(\mathrm{Hz})\end{array}$ & $\begin{array}{c}\text { Freq. range } \\
(\mathrm{Hz})\end{array}$ & $\begin{array}{c}\text { Sampling rate } \\
(\mathrm{Hz})\end{array}$ & $\begin{array}{c}\text { Resolution } \\
(\mathrm{bit})\end{array}$ \\
\hline ECG (per lead) & $0.01 \ldots 60-125$ & $120-250$ & 16 \\
\hline Temperature & $0 \ldots 0.1-1$ & $0.2-2$ & 12 \\
\hline Oximetry & $0 \ldots 30$ & 60 & 12 \\
\hline Arterial pressure & $0 \ldots 60$ & 120 & 12 \\
\hline Respiration rate & $0.1 \ldots 10$ & 20 & 12 \\
\hline Cardiac rate & $0.4 \ldots 5$ & 10 & 12 \\
\hline
\end{tabular}

TABLE II

ALERT DETECTION PARAMETERS

\begin{tabular}{|c|c|}
\hline Alert Type & Detection Parameter \\
\hline low $\mathrm{SpO}_{2}$ & $\mathrm{SpO}_{2}<90 \%$ \\
\hline bradycardia & $\mathrm{HR}<40 \mathrm{bpm}$ \\
\hline tachycardia & $\mathrm{HR}>150 \mathrm{bpm}$ \\
\hline HR change & $|\Delta \mathrm{HR} / 5 \mathrm{~min}|>19 \%$ \\
\hline HR stability & max HR variability from past 4 reads $>10 \%$ \\
\hline BP change & systolic or diastolic change $> \pm 11 \%$ \\
\hline
\end{tabular}

It should be noted that at non-emergency medical situations, ECG and $\mathrm{SpO} 2$ signals are usually transmitted in bursts, while signals such as body temperature and blood glucose, are transmitted in a single packet to the base-station [6]. In fact, to reduce the traffic load and the power consumption of a BSN, the current trend in telemedicine systems is to enhance sensor node intelligence, available memory, processing power, and only enable on-line solicited requests for results. In this way, continuous and bulky data transfer is rather seldom, occurring only in intermittent occasions [6]. However in emergency cases this should not be the rule, since patient's life is priceless and above to any other kind of considerations. Continuous and bulky data transfer in real-time might be prevalent here.

\section{QOS NEEDS IN E-HEALTH}

Some authors argue that differentiation based in data priority is inherent to wireless sensor networks (WSN), since it is normal to have sensors to monitor distinct physical parameters simultaneously, just as happens in BSNs. Here, the importance of the collected information is necessarily distinct, and therefore the network must prioritize the transmission of critical data when occurs a sudden clinical change in the patient. For example, in patients with cardiac diseases, heart activity information is more important than body temperature data. And depending on the patient's clinical condition, the priority assigned to a vital signal can change dynamically. For instance, glucose data might be assigned a low priority when the readings are in normal range, but a higher priority might be reassigned to it when readings indicate hypo or hyper-glycemia.

Most current BSNs offer only best-effort service [7], which is not the most adequate for e-emergency. The given examples indicate that those networks require QoS support in critical cases. This would ensure the adequate bandwidth to higher priority streams for an efficient data delivery, even in case of interference or fading.

QoS mechanisms are usually deployed in networks to guarantee consistent service levels concerning certain parameters, such as, packet loss ratio, transmission delay, jitter, and available bandwidth. These are the traditional endto-end parameters used to characterize the performance of any communication infrastructure, including BSNs. For instance, the total delay to an ECG signal being displayed in the monitor should be less than $3 \mathrm{~s}$ for useful real time analysis by the cardiologists [8]. And to guarantee that jitter does not affect the estimation of the R-wave fiducial point, which modifies considerably the spectrum, ECG signals require a minimum sampling rate of $250 \mathrm{~Hz}$ [9]. It should be noted that no significant difference between ECG traces are detected by sampling the signal at rates between 250 and 500 $\mathrm{Hz}$; but significant reduction in peak amplitude values and inaccurate interval measurements are obtained at 125 samples/s [6].

However, QoS in BSNs may not be fully described using only those parameters, because of its context aware nature. For example, QoS at application level may be regarded as guaranteeing the right number of sensors for monitoring the vital signals according to the patients' emergency state.

The available energy in the BSN is another very important parameter to have into account. In fact, if energy is carelessly consumed, the BSN may rapidly become completely useless by lack of power. To prevent such failure, energy should be carefully saved using different approaches. For example, if the patient is in normal state then the sampling rate of sensors can be reduced to save power, or if the battery charge becomes low then its energy should be reserved to the more vital tasks of the patient. That is to say, the monitoring activity should adapt in accordance with the patient clinical state for energy saving. It should be realized that to save further energy, communication protocols should be simple, and data should be aggregated, compressed, and transmitted in loaded packets, since computing demands much less energy than transmission. However, attention must be paid to the delay, since it tends to increase linearly with the packet length.

Other distinct ways than prioritizations may be used additionally to provide QoS. For example, for efficiency reasons a large packet length is chosen for non-critical situations. But as soon as an emergency occurs, the packet size is reduced to meet the low delay QoS requirement, and signals considered irrelevant to this emergency episode are sampled at a lesser rate, or not sampled at all.

Moreover, the computation power may be lowered to a minimum since all the data must be forwarded, in opposition to the regular operation where, to save energy, the cardiorespiratory rhythm can be computed on-board before sending it. Or else, an ECG signal could be processed in the sensor itself to extract its relevant features. In this way, only 
information about an event is transmitted (e.g., QRS features and the corresponding timestamp of R-peak), hence reducing the traffic load and saving energy.

A BSN does not transmit only measurement data packets. Other packets may be present, such as those carrying control or alerting data. In this way, it is suggested that a high priority level should be assigned to data packets carrying alarming notification and measurements, and acknowledgement of correctly received packets; medium priority level should be assigned to scheduled transmissions of data packets, and primary control packets (e.g. sensor configuration); low priority level should be given to periodic polling of the nodes for network integrity check, and secondary control packets (e.g. link) [6].

The vital signals captured from the patient body must be delivered to a remote diagnosis and supporting centre, through some available communication infrastructure, such as the Internet or a $3 \mathrm{G}$ mobile communication network. As a result, the delivered QoS depends necessarily on the network infrastructure chosen for the delivery of mobile health services. Therefore, to meet the required QoS, the mobile health services platform needs to be able to acquire and use contextual information about the QoS offered by communication network infrastructures available at the patient's current location and time [7]. High availability and reliability are the most desirable characteristics that these network infrastructures should offer, as well as QoS guarantees for bandwidth, end-to-end delay, jitter and loss.

\section{E-HEALTH WIRELESS SYSTEMS WITH QOS}

Despite the number of healthcare systems already developed [1], only few encompass QoS requirements. In order to assess how QoS support is being deployed in healthcare sensor networks, some projects were analyzed, as well as the QoS requirements that the respective authors have considered important to incorporate in their implementations. Based on the related literature, QoS projects for e-health can be grouped according to the following topics: A) frameworks with QoS; B) QoS through reconfiguration; C) algorithms for QoS enhancement.

\section{A. Frameworks with $Q o S$}

She et al. [10] propose an infrastructure for remote medical applications using ZigBee and commercial $3 \mathrm{G}$ networks. In order to improve the delay and the transmission time of critical vital signals, so improving the overall QoS in terms of latency, bandwidth, and power consumption, a differentiated service based on priority scheduling and data compression is presented. For example, for a cardiac disease patient, a higher priority level is assigned to ECG signals than to body temperature. Thus ECG signals will be processed and sent earlier than body temperature if both arrive at the personal server at the same time. High data rate and delay tolerant signals will be compressed and stored in local memory for later transmission. The physician analyses the incoming data and will act accordingly to the clinical episode.

Vergados et al. [11] propose a wireless DiffServ infrastructure for mobile telemedicine. Formed by several ehealth DiffServ domains, the network can reliably handle both normal and life-critical medical applications under extreme traffic conditions. Assigning different priority levels according to the specific medical application requirements, and according to the urgency of the medical incident, causes the network to intelligently drop and/or delay the packets, in order to achieve a high service level.

\section{B. QoS through Reconfiguration}

Usually a WSN is reconfigured based on common parameters, such as traffic load, node failures, channel utilization, energy drainage, etc. Gondal et al. [3] suggest that the reconfiguration also takes into account the physician's recommendations for patient monitoring schedule, the condition of the physiological parameters being sensed by the network, and the disease diagnosis outcome from an automated or manual system. This information will be fed back into the body area network so that it can selfreconfigure to monitor the patient with the required intensity, while concomitantly tries to maximize the network reliability, throughput and lifetime. This operation of translating the clinical operations into network sensing schedules and reconfiguration decision, providing in this way a service with the required monitoring quality, is the key focus of this framework.

MiLAN [12] is a middleware system targeted for reconfiguring centralized networks with few sensors and no mobility, particularly BSNs. It assumes that a vital signal may be read from different sensors with different reliabilities. For instance, ECG and $\mathrm{SpO} 2$ sensors can provide heart rate with reliability of 1 and 0.7 , respectively. In this way, MiLAN uses graphs provided by the application, together with information about the current application state, to decide how to configure and manage both the network and sensors in order to meet the QoS application's requirements, e.g. the reading of a vital signal with a defined reliability. At the same time, it tries to maximize the application lifetime, instead of the sensors lifetime. However this kind of approach seems unsuitable for e-emergency systems, as full reliability is required.

\section{Algorithms for QoS Enhancement}

The common way to recover the lost or corrupted data in connectionless transmissions is through retransmission processes. However common automatic requests mechanisms are unable to guarantee data recovering in a bounded transmission delay, as required in e-emergency systems. To tackle this problem, Henrion et al. [13] use restoration algorithms to recover the ECG missing packets that do not arrive to the monitoring equipment within an acceptable delay. Simulations results showed that, even for $8 \%$ of packet loss in transmission of ECG data during $30 \mathrm{~s}$, the 
restoration scheme allow reconstructing a more functional signal for a medical expertise.

Coelho Jr. et al. [14] apply the concept of QoS to power management in a real-time remote physiological monitoring system. The authors present a power management model so that an application can adapt dynamically to particular situations, generating less requests from the devices, and therefore saving energy. The model is based on an extended power state machine. In the case of ECG monitoring, examples of possible states can be a patient with a normal ECG, a patient with low risk abnormalities, and a patient with high risk abnormalities; examples of events can be loss of communication channel, ECG abnormal for the last five minutes, or patient signaling that is not feeling well.

Table III presents an overview of the discussed projects regarding the QoS aspects that the authors have considered important to benefit their systems with, as well as the target environments where projects are intended to be deployed.

TABLE III

OVERVIEW OF THE DISCUSSED HEALTHCARE SYSTEMS

\begin{tabular}{|c|c|c|c|}
\hline & Description & QoS Goals & Target Deployment \\
\hline $\begin{array}{l}\text { She et } \\
\text { al. }\end{array}$ & $\begin{array}{l}\text { DiffServ based on } \\
\text { transmission } \\
\text { priority } \\
\text { scheduling, data } \\
\text { compression }\end{array}$ & $\begin{array}{l}\text { low delay and } \\
\text { loss rate for } \\
\text { vital signals, } \\
\text { better } \\
\text { bandwidth } \\
\text { utilization }\end{array}$ & $\begin{array}{l}\text { remote medical } \\
\text { applications using } \\
\text { Zigbee and } \\
\text { commercial 3G } \\
\text { networks }\end{array}$ \\
\hline $\begin{array}{l}\text { Verga- } \\
\text { dos et } \\
\text { al. }\end{array}$ & $\begin{array}{l}\text { wireless DiffServ } \\
\text { infrastructure }\end{array}$ & $\begin{array}{l}\text { low loss rate, } \\
\text { guaranteed } \\
\text { bandwidth }\end{array}$ & $\begin{array}{l}\text { reliable network for } \\
\text { mobile telemedicine } \\
\text { under extreme traffic } \\
\text { conditions }\end{array}$ \\
\hline $\begin{array}{l}\text { Gondal } \\
\text { et al. }\end{array}$ & $\begin{array}{l}\text { send feedback } \\
\text { information into } \\
\text { BSN to self- } \\
\text { reconfiguration }\end{array}$ & $\begin{array}{l}\text { application } \\
\text { level } \\
\text { improvement }\end{array}$ & $\begin{array}{c}\text { remote and local } \\
\text { patient monitoring }\end{array}$ \\
\hline MiLAN & $\begin{array}{l}\text { middleware } \\
\text { system for } \\
\text { reconfiguring } \\
\text { BSNs }\end{array}$ & $\begin{array}{l}\text { application } \\
\text { level } \\
\text { improvement }\end{array}$ & $\begin{array}{c}\text { increase BSN lifetime } \\
\text { while respecting the } \\
\text { QoS needs }\end{array}$ \\
\hline $\begin{array}{l}\text { Henri- } \\
\text { on et al. }\end{array}$ & $\begin{array}{l}\text { restoration } \\
\text { algorithms }\end{array}$ & $\begin{array}{l}\text { recover } \\
\text { missing } \\
\text { packets }\end{array}$ & $\begin{array}{l}\text { reconstruct a more } \\
\text { functional signal for a } \\
\text { medical expertise }\end{array}$ \\
\hline $\begin{array}{l}\text { Coelho } \\
\text { et al. }\end{array}$ & $\begin{array}{c}\text { power } \\
\text { management } \\
\text { model based on } \\
\text { application states }\end{array}$ & energy saving & $\begin{array}{l}\text { reserve power to the } \\
\text { patient's more vital } \\
\text { tasks }\end{array}$ \\
\hline
\end{tabular}

\section{CONCLUSIONS}

Since patient's life is priceless, emergency healthcare networks should be totally reliable and efficient. Therefore these networks must support QoS as they demand clearly for reliability, guaranteed bandwidth, and low delays due to their real-time nature.

Despite the number of healthcare systems already developed, only few address QoS support. According to the representative wireless e-health projects herein surveyed, it was observed that QoS support provided in each approach is varied and targets different QoS levels for specific uses. In order to better compare the studied QoS approaches, a summary including their main characteristics and goals is herein provided. However, the QoS support and deployment levels offered by these systems are not simple or satisfactory enough to fulfill an expected need in hospital units: providing a wireless, pervasive, valuable and totally reliable assistance to any patient with health risk abnormalities.

\section{REFERENCES}

[1] E. Kyriacou et al., "e-Health e-Emergency Systems: Current Status and Future Directions", IEEE Antennas \& Propagation Mag., 49(1), 2007.

[2] T. Gao et al., "Vital Signs Monitoring and Patient Tracking Over a Wireless Network", Proc. of the 27th IEEE EMBS, Sept. 2005.

[3] I. Gondal et al., "Integrated sensing and diagnosis - the next step in real time patient health care", 6th IEEE/ACIS ICIS, Melbourne, 2007.

[4] M. Paksuniemi et al., "Wireless sensor and data transmission needs and technologies for patient monitoring in the operating room and intensive care unit", Proc. 27 $7^{\text {th }}$ IEEE EMBC, pp.5182-5185, Shanghai, Sept. ' 05.

[5] Arnon et al., "A comparative study of wireless communication network configurations for medical applications" IEEE Wirel. Comm. $10(1),{ }^{\prime} 03$.

[6] I. Lamprinos et al., "Communication protocol requirements of patient personal area nets. for telemonitoring", Tech. \& Health Care, 14( $3), 06$.

[7] . Wac et al., "Context-aware QoS Provisioning in an m-health Services Platform", Inter. Journal of IP Tech., 2 (2) pp.102-108, 2006.

[8] A. Iglesias et al., "Performance Study of Real-Time ECG Transmission in Wireless Networks," ITAB'06, Ioannina, Greece, 2006.

[9] Pinna et al., "The accuracy of power-spectrum analysis of heart-rate variability from annotated RR list generated by Holter systems", Physiol. Meas., n.15:163-179, 1994.

[10] H. She et al., "A Network-based system architecture for remote medical applications", Asia-Pacific Advanced Network, China, Aug. 2007.

[11] Vergados et al., "Applying Wireless DiffServ for QoS Provisioning in Mobile Emergency Telemedicine", IEEE GLOBECOM, Nov. 2006.

[12] W. B. Heinzelman et al., "Middleware to support sensor network applications", IEEE Network, 18(1):6-14, 2004.

[13] S. Henrion et al., "Transmitting critical biomedical signals over unreliable connexionless channels with good QoS using advanced signal processing", proc. of WSEAS, pp. 694-700, Athens, Greece, Jan. 2004.

[14] Coelho Jr. et al., "A biomedical wearable device for remote monitoring of physiological signals", Proc. IEEE ETFA, v. 2:708713 , Sept., 2003.

[15] T. R. F. Fulford-Jones et al., "A Portable, Low-Power, Wireless TwoLead EKG System", Proceedings of the 26th IEEE EMBS, Sept. 2004.

[16] N. Chevrollier et al., "On the use of wireless network technologies in healthcare environments", proc. $5^{\text {th }}$ IEEE AWSN workshop, Jun. ' 05.

[17] N. F. Timmons et al., "Analysis of the performance of IEEE 802.15.4 for medical sensor body area networking", proc. IEEE SECON, Oct.'04. 